\title{
Function-Theoretic Explanation and the Search for Neural Mechanisms
}

\author{
Frances Egan
}

\section{Introduction}

A common kind of explanation in cognitive neuroscience might be called functiontheoretic: with some target cognitive capacity in view, the theorist hypothesizes that the system computes a well-defined function (in the mathematical sense) and explains how computing this function constitutes (in the system's normal environment) the exercise of the cognitive capacity. Recently, proponents of the so-called 'new mechanist' approach in philosophy of science have argued that a model of a cognitive capacity is explanatory only to the extent that it reveals the causal structure of the mechanism underlying the capacity. If they are right, then a cognitive model that resists a transparent mapping to known neural mechanisms fails to be explanatory. I argue that a functiontheoretic characterization of a cognitive capacity can be genuinely explanatory even absent an account of how the capacity is realized in neural hardware.

\section{Function-Theoretic Explanation}

Marr's (1982) theory of early vision purports to explain edge detection by positing the computation of the Laplacian of a Gaussian of the retinal array. The mechanism takes as input intensity values at points in the image and calculates the rate of intensity change over the image. In other words, it computes a particular smoothing function. Ullman (1979) hypothesizes that the visual system recovers the 3D structure of moving objects by computing a function from three distinct views of four non-coplanar points to the unique rigid configuration consistent with the points. Shadmehr and Wise's (2005) computational account of motor control putatively explains how a subject is able to grasp an object in view by computing the displacement of the hand from its current location to the target location, i.e. by computing vector subtraction. In a well-known 
example from animal cognition, Gallistel (1990) purports to explain the Tunisian desert ant's impressive navigational abilities by appeal to the computation of the displacement vector to its nest from any point along its foraging trajectory. Seung et al. $(1996,1998,2000)$ hypothesize that the brain keeps track of eye movements by deploying an internal integrator.

These examples illustrate an explanatory strategy that is pervasive in computational cognitive science. I call the strategy function-theoretic explanation and the mathematical characterization that is central to it function-theoretic characterization. ${ }^{1}$ (Henceforth, I shall abbreviate "function-theoretic" as FT.) Theories employing the strategy explain a cognitive capacity by appeal to an independently well-understood mathematical function under which the physical system is subsumed. In other words, what gets computed, according to these computational models, is the value of a mathematical function (e.g., addition, vector subtraction, the Laplacian of a Gaussian, a fast Fourier transform) for certain arguments for which the function is defined. For present purposes we can take functions to be mappings from sets (the arguments of the function) to sets (its values). A fully specified theory of a cognitive capacity will go on to propose an algorithm by which the computation of the value of the function(s) is effected, and describe the neural hardware that implements the computation. ${ }^{2}$

A function-theoretic description provides a domain-general, environment-neutral characterization of a mechanism. It prescinds not only from the cognitive capacity that is the explanatory target of the theory (vision, motor control, etc.), but also from the environment in which the capacity is normally exercised. In fact, the abstract nature of the FT characterization - in particular, the fact that as an independently characterized mathematical object the function can be decoupled from both the environmental context and the cognitive domain that it subserves-accounts for perhaps the most significant explanatory virtue of function-theoretic characterization. The mathematical functions deployed in computational models are typically well understood independently of their use in such models. Laplacian of Gaussian filters, fast Fourier transforms, vector subtraction, and so on are standard items in the applied mathematician's toolbox. To apply one of these tools to a biological system - to subsume the system under the mathematical description-makes sense of what might otherwise be a heterogeneous collection of input-output pairs. ("I see what it's doing... it's an integrator!") 3 And

\footnotetext{
1 This sense of function-theoretic characterization is not to be confused with various notions of functional explanation in the literature, in particular, with Cummins' (1975) notion of functional analysis. However, a functional analysis of a complex system may involve function-theoretic characterization, in the sense explicated in this chapter.

2 The FT characterization, the specification of the algorithm, and the neural implementation correspond, roughly, to Marr's three levels of description-the computational, algorithmic, and implementation, respectively. The topmost, computational, level of theory also adverts to general environmental facts ('constraints') essential to the explanation of the cognitive capacity, as discussed below. See Egan (1995) for elaboration and defense of this account of Marr's computational level.

${ }^{3}$ Moreover, theorists typically have at their fingertips various algorithms for computing these functions. Of course, the algorithms are hypotheses that require independent support, but the point is that the theorist has a ready supply of such hypotheses.
} 
since the FT characterization specifies the function intensionally, typically in terms of an algorithm for computing the function, it provides the basis for predicting the output of the device in a wide range of circumstances that go well beyond the observed data set.

But, of course, the theorist of cognition must explain how computing the value of the specified function, in the subject's normal environment, contributes to the exercise of the cognitive capacity that is the explanatory target of the theory-for the motor control mechanism, the capacity to grasp an object in nearby space, for visual mechanisms, the capacity to see "what is where" (as Marr puts it) in the nearby environment. Only in some environments would computing the Laplacian of a Gaussian help an organism to see. In our environment this computation produces a smoothed output that facilitates the detection of sharp intensity gradients across the retina, which, when these intensity gradients co-occur at different scales, correspond to physically significant boundaries-changes in depth, surface orientation, illumination, or reflectance-in the scene. Ullman's structure-from-motion mechanism succeeds in recovering the $3 \mathrm{D}$ structure of a moving object by computing the unique rigid configuration consistent with three distinct views of four non-coplanar points on the object only because, in our world, most objects are rigid in translation (the rigidity assumption). Thus, to yield an explanation of the target cognitive capacity, the environment-neutral, domaingeneral characterization given by the FT description must be supplemented by environment-specific facts that explain how computing the value of the specified mathematical function, in the subject's normal environment, contributes to the exercise of the target cognitive capacity.

One way to connect the abstract FT characterization to the target cognitive capacity is to attribute representational contents that are appropriate to the relevant cognitive domain. Theorists of vision construe the mechanisms they posit as representing properties of the light, e.g., light-intensity values, changes in light intensity, and, further downstream, changes in depth and surface orientation. The inputs and outputs of the Laplacian/Gaussian filter represent light intensities and discontinuities of light intensity, respectively. Theorists of motor control construe the mechanisms they posit as representing positions of objects in nearby space and changes in joint angles. But the fact that a mechanism characterized function-theoretically can also be characterized in terms of representational contents appropriate to the cognitive domain in question does not obviate the explanatory interest of the more abstract, domain-general, mathematical characterization that is the focus of this chapter. ${ }^{4}$

I will have much more to say about function-theoretic explanation as we progress, but I have said enough to set up the main issue of the chapter. I turn now to the challenge from the new mechanists.

\footnotetext{
${ }^{4}$ In Egan (2014) I argue that representational contents are best construed as part of an explanatory gloss on a computational theory, that they serve a variety of pragmatic purposes but are, strictly speaking, theoretically optional. The argument in this chapter does not depend on any particular view of representational content. (Though see the postscript.)
} 


\section{The New Mechanistic Philosophy}

A mechanism is an object that performs some function in virtue of the operations of its component parts and their organization. Mechanistic explanation is the explanation of the capacities of a system by reference to the properties and operations of its component parts and their causal organization. ${ }^{5}$

Proponents of the new mechanistic philosophy claim that all genuine explanation in cognitive neuroscience is mechanistic explanation:

The common crux of mechanistic explanation, both in its current form and in forms stretching back through Descartes to Aristotle, is to reveal the causal structure of a system. Explanatory models are counted as good or bad to the extent that they capture, even dimly at times, aspects of that causal structure. (Piccinini and Craver 2011, 292)

Explanations in computational neuroscience are subject to precisely these same norms [the norms of mechanistic explanation]. The cost imposed by departing from this view... is the loss of a clear distinction between computational models that genuinely explain how a given phenomenon is actually produced versus those that merely describe how it might possibly be produced... And it is precisely by adhering to this distinction (along with a distinction between merely describing or saving a phenomenon and explaining it), that one can identify models in computational neuroscience possessing explanatory force. (Kaplan 2011, 346)

Levy (2013) provides a useful gloss on the view that he calls explanatory mechanism: "to understand a phenomenon one must look under the hood and discern its underlying structure" (107). The idea that a cognitive model has explanatory force just to the extent that it reveals the causal structure of an underlying mechanism is explicated in terms of what Kaplan calls a model-mechanism-mapping (3M) constraint on explanatory models:

(3M) A model of a target phenomenon explains that phenomenon to the extent that (a) the variables in the model correspond to identifiable components, activities, and organizational features of the target mechanism that produces, maintains, or underlies the phenomenon, and (b) the (perhaps mathematical) dependencies posited among these (perhaps mathematical) variables in the model correspond to causal relations among the components of the target mechanism. (Kaplan 2011, 347; see also Kaplan and Craver 2011, 611)

The $3 \mathrm{M}$ Constraint is claimed to distinguish genuine explanations in cognitive neuroscience from mere descriptions and predictive devices (Kaplan 2011, 340). There is no doubt that many explanatory models in cognitive neuroscience do conform to the new mechanists' strictures. But if the $3 \mathrm{M}$ Constraint is a necessary condition on genuine explanation, then many promising cognitive models turn out not to be explanatory. ${ }^{6}$

\footnotetext{
${ }^{5}$ For characterization of mechanisms and mechanistic explanation see Bechtel (2008); Bechtel and Abrahamsen (2005); Bechtel and Richardson (1993); Craver (2006, 2007).

${ }^{6}$ Kaplan intends the $3 \mathrm{M}$ Constraint to be both necessary and sufficient for genuine explanation: "A central tenet of the mechanistic framework is that the model carries explanatory force to the extent that it reveals aspects of the causal structure of a mechanism, and lacks explanatory force to the extent
} 
In Sections 4 and 5 I argue that FT models often do not fit the mechanists' account, yet they can be, and often are, genuinely explanatory.

\section{Function-Theoretic Models and Mechanistic Explanation}

Satisfying the $3 \mathrm{M}$ Constraint requires a decomposition of a cognitive system into components. How is this crucial notion to be understood? According to Craver (2007) "[c] omponents are the entities in a mechanism - what are commonly called 'parts" " (128). He goes on to characterize the relationship between mechanisms and their components as follows:

Organization is the inter-level relation between a mechanism as a whole and its components. Lower-level components are made up into higher-level components by organizing them spatially, temporally, and actively into something greater than a mere sum of the parts. (Craver 2007, 189)

It follows that an entity cannot be a component of itself. Moreover, the components (parts) of the system should be distinct structures, or at least, not characterized simply in functional terms, on pain of trivializing the mechanists' requirement on genuinely explanatory accounts in neuroscience. ${ }^{7}$ As Piccinini and Craver (2011) note, the point of mechanistic explanation is to reveal the causal structure of a system; this requires that the components (parts) over which causal transitions are defined be understood as structures of some sort.

Let's consider the two parts of the $3 \mathrm{M}$ Constraint in turn. Condition (a) requires that variables in the model correspond to components, activities, and organizational features of the target neural mechanism. In the case of FT models the variables range over the arguments and values of a mathematical function. But often, perhaps even typically, nothing in the function-theoretically characterized system corresponds to (states of) components of neural mechanisms. ${ }^{8}$ The Marrian filter computes a function from intensity values at points in the image to the rate of intensity change over the image. The Ullman structure-from-motion system calculates the unique rigid structure compatible with three distinct views of four non-coplanar points. The presumption, of course, is that these mathematically characterized systems are realized in neural hardware, but in neither case is the implementing hardware specified at the level of its component parts and their organization. Shadmehr and Wise (2005) hypothesize that

that it fails to describe this structure" (2011, 347). I am not here challenging the sufficiency claim. Revealing the causal structure of a mechanism often is explanatory when we wish to understand how a particular effect occurs. The point is that the constraint does not account for the explanatory force of an important class of cognitive models.

${ }^{7}$ Milkowski $(2013,55)$ argues that a mechanistic analysis must "bottom out" in the constitutive level, the level at which "the structures that realize the computation are described."

${ }^{8}$ Since the relevant variables in FT models are mathematical, the $3 \mathrm{M}$ constraint should be interpreted as requiring a mapping from values of the variables to states of component parts. This is how I will understand the constraint. 
the motor control system that computes vector subtraction is realized in a network in the pre-motor cortex, but again, nothing in the FT characterization corresponds to (states of) components of the network and their organization. ${ }^{9}$ And Seung et al.s (1996, 1998, 2000) model of oculomotor control posits an internal integrator without specifying any correspondence between variables over which the computation is defined and (states of) components of implementing neural hardware. I discuss this example in more detail below.

Let us turn now to condition (b) of the $3 \mathrm{M}$ Constraint, which requires that the dependencies among the variables in the model correspond to causal relations among components of the target neural mechanism. In the case of FT models the dependencies among the variables ranging over the arguments and values of the specified function are, of course, mathematical. The presumption that systems characterized in FT terms are realized in neural hardware-as they must be if the FT model is to be true of the organism - amounts to the idea that there exists a mapping from physical state-types to the arguments and values of the specified mathematical function, such that causal state transitions among the physical states are interpreted as mathematical relations among the arguments and values of the function. A complete computational

\footnotetext{
${ }^{9}$ I am not denying that computational theorists sometimes attempt to specify correspondences between variables in their models and (states of) components of neural mechanisms. In explaining forward kinematics - the computation of target location in body-centered coordinates from information about eye orientation and retinotopic location of target-Shadmehr and Wise appeal to Zipser and Anderson's (1988) three-layer neural network model of gain modulation. Some nodes in the model's input layers represent (correspond to) eye orientation and others retinotopic location of target; output layers represent (correspond to) target location in body-centered coordinates. Zipser and Anderson hypothesized that neurons in area LIP and area 7A in the parietal cortex play the relevant computational roles. It is not implausible, then, to describe input and output layers in the Zipser-Anderson model as component parts of a neural mechanism. Interestingly, the Zipser-Anderson models fails to count as genuinely explanatory by Kaplan's lights. He says:
}

The real limitations on the explanatory force of the Zipser-Andersen model is that it is difficult if not impossible to effect a mapping between those elements in the model giving rise to gain-modulated hidden unit activity and the neural components in parietal cortex underlying gain-modulated responses (arguably, the core requirement imposed by $3 \mathrm{M}$ on explanatory mechanistic models). (Kaplan 2011, 365-6)

Kaplan cites two reasons why the model fails to be genuinely explanatory: First, it is difficult in general to effect a mapping between neural network models and target neural systems. There is typically only a loose and imprecise correspondence between network architecture and neural implementation (see, e.g., Crick 1989; Smolensky 1988; Kaplan 2011, 366). Secondly, Kaplan notes that there are competing models of how gain modulation is implemented in the brain, each enjoying some empirical support, and so, he concludes, the Zipser-Anderson model is just a "how possibly" model and not genuinely explanatory.

An interesting question is whether, according to mechanists, the apparent failure of the Zipser-Anderson model to satisfy the $3 \mathrm{M}$ Constraint thereby undermines the explanatory bona fides of the Shadmehr-Wise function-theoretic model that it is supposed to implement. Presumably it does, since variables in the high-level characterization do not in fact correspond to components in an explanatory model of neural mechanisms. A consequence of the mechanist constraint would seem to be that any breakdown or lacunae in the decomposition (all the way down to basic physical constituents?) would threaten the explanatory credentials of higher-level theories. According to Kaplan (personal correspondence) the $3 \mathrm{M}$ constraint requires only that some variables are mapped to components, thus allowing for partial or incomplete explanations. 


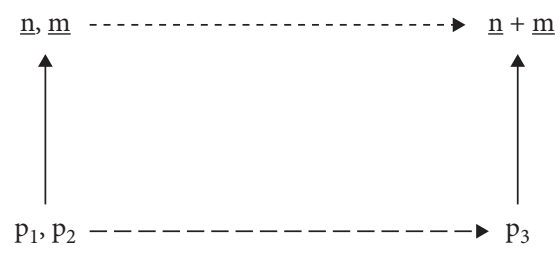

Figure 7.1 An adder

explanation of a cognitive capacity will specify this mapping. Consider the characterization of a device that computes the addition function (Figure 7.1).

A physical system computes the addition function just in case there exists a mapping from physical state types to numbers, such that physical state types related by a causal state-transition relation $\left(\left(\mathrm{p}_{1}, \mathrm{p}_{2}\right) \rightarrow \mathrm{p}_{3}\right)$ are mapped to numbers $\mathrm{n}, \mathrm{m}$, and $\mathrm{n}+\mathrm{m}$ related as addends and sums. Whenever the system goes into the physical state specified under the mapping as $\mathrm{n}$, and then goes into the physical state specified under the mapping as $\mathrm{m}$, it is caused to go into the physical state specified under the mapping as $\mathrm{n}+\mathrm{m}$.

It follows that the function-theoretic description provides an abstract characterization of causal relations among initial and end states of the realizing physical mechanism, whatever that happens to be. The physical states $\left[p_{1} \ldots p_{n}\right]$ that are characterized as the arguments and values of the function (as addends and sums in the above example) in the complete computational model may count as components (in the sense explicated above) of the neural mechanism, but there is no reason to assume that they must. In precisely those cases where condition (a) of the $3 \mathrm{M}$ constraint fails to be satisfiedwhere the arguments and values of the specified function do not correspond to (states of) components of the neural mechanism-condition (b) will fail to be satisfied as well-the dependencies among the variables specified by the mathematical description will not correspond to causal relations among (states of) components of the target neural mechanism.

Seung's $(1996,1998)$ model of oculomotor memory illustrates this failure. It is worth examining the case in more detail.

The last forty years has seen a good deal of experimental and theoretical work on oculomotor control. ${ }^{10}$ Saccadic eye movements shift the eyes rapidly from one position in the visual field to another. Between saccades the eyes remain stationary; experimental results show that normal humans can hold their eyes still at arbitrary positions for twenty or more seconds, even in complete darkness (Becker and Klein 1973; Hess et al. 1985). The most plausible explanation is that the brain maintains current eye position after a stimulus has gone by employing a short-term memory of eye positions. The experimental data support the hypothesis of a multi-stable recurrent network located in the brainstem that takes as input transient eye velocities and gives as output

${ }^{10}$ For general discussion see Robinson 1989, Glimcher 1999, and Leigh and Zee 2006. 
persistent eye positions (see Seung 1996, 1998). It does so by accumulating input pulses, adding or subtracting (depending on the direction of movement) new inputs from the previous summation. In other words, it performs integration. A second, "read-out" network reads the current position and stabilizes the eye by controlling the length-tension relationships of the muscles. Seung (1998) describes the neural integrator as follows:

In the oculomotor system, the integrator can be regarded as an internal model. The location of this internal model is known, unlike in other motor systems. As a steady eye position can be maintained without proprioceptive or visual feedback, the quality of the internal model is very good. And physiological studies of this internal model indicate that it is a recurrent neural network with a continuous attractor. (Seung 1998, 1257)

Encoding eye position in neural activity requires a continuous, analog-grade code, thus motivating the choice of a continuous attractor network. ${ }^{11}$ Figure 7.2 illustrates the continuous line attractor dynamics of the network. A new stimulus changes the state of the network away from a line of fixed points. The network then settles on a new point along the attractor line; this point encodes the current eye position. Line attractor neural networks are posited to underlie a wide variety of motor control functions. ${ }^{12}$

Two features of the neural integrator are of special interest for present purposes. In the first place, the network has no proprietary input and output units; each unit is interconnected to all other units and can receive external stimuli (that is, pulse saccades). Secondly, no single unit or proper subset of total units represents an eye position; rather, only the total state of the network at a given time is a candidate for encoding a persistent eye position. Points in the state-space portrait (Figure 7.2) do not represent the activity of single cells, but rather the collective activity of the whole network. At any given moment the network "occupies" a point in the portrait, and it "aspires to" the line attractor. Points along the line attractor are collective states of the network that represent persistent eye positions.

Let's consider these two features of the network in light of the mechanists' $3 \mathrm{M}$ constraint. The computation effected by the integrator takes as arguments eye-movement velocities and gives as values persistent eye positions. Condition (a) of the $3 \mathrm{M}$ constraint, recall, requires that the variables in the model "correspond to identifiable components, activities, and organizational features of the... mechanism that... underlies the phenomenon" (Kaplan 2011, 347). Arguably, neither variable of the FT model corresponds to (states of) components of the network that realizes the computation. With respect to the arguments of the function: as noted above, any of the networks' units can receive external stimuli from pulse saccades generating eye velocities. The mechanist may respond that eye velocities correspond to (are realized by) states of components of

\footnotetext{
${ }^{11}$ See Seung $(1996,1998)$. For general discussion of attractor networks see Amit (1989), Eliasmith and Anderson (2003), and Eliasmith (2005).

12 Besides Seung (1996) and Seung et al. (2000), see Shadmehr and Wise (2005).
} 


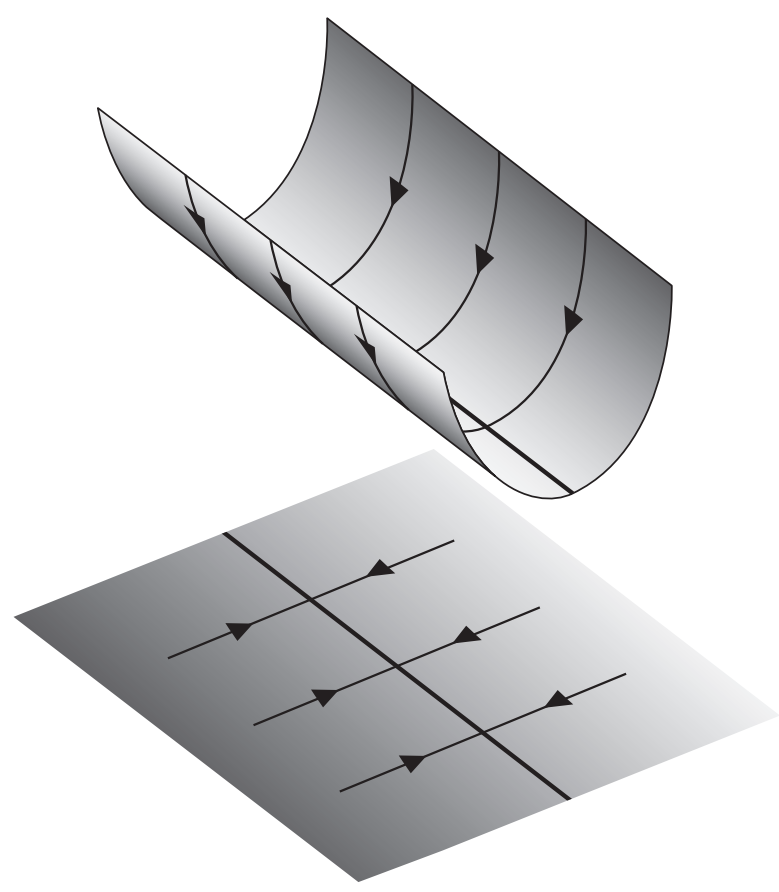

Figure 7.2 A state-space portrait for the eye-position memory network. All trajectories in the state space of the network flow toward a line attractor (thick line). Each point in the line is a persistent state that represents a different eye position.

Source: Seung (1996), 13340. Reprinted with permission of the National Academy of Sciences, U.S.A.

the mechanism; they correspond to states of different components for each run of the computation. But the mechanist cannot take this line for the values of the function. Persistent eye positions correspond to (are realized by) collective states of the whole network. On any plausible construal of "component," collective states of the whole network do not count as components of the network. And while it is certainly true that the values of the integration do correspond to (are realized by) "activities" and "organizational features" of the network, a weakened construal of condition (a) of the $3 \mathrm{M}$ constraint that makes no mention of components and their interactions amounts to nothing more than the requirement that the model is realized by neural hardware; in other words, it imposes only the requirement that there exist a mapping of the sort depicted in Figure 7.1. ${ }^{13}$

\footnotetext{
${ }^{13}$ Bechtel and Richardson (1993) discuss neural network models where "classical mechanistic strategies-and in particular, decomposition and localization-fall short" (203). They leave open whether an "explanatory strategy that abandons localization and decomposition... constitutes a properly mechanistic approach" (203). I want to leave this issue open too. There is no question that the behavior of the neural integrator is a function of the interaction of its parts. Thus an account of the operation of the integrator would be mechanistic in some intuitive sense. But the $3 \mathrm{M}$ Constraint, which requires a transparent
} 
Turning to condition (b) of the $3 \mathrm{M}$ constraint: since condition (a) is not satisfied-the arguments and values of the function do not correspond to (states of) components of the neural mechanism-condition (b) is not satisfied either. While the FT model gives an abstract characterization of causal relations between initial and end states of the attractor network, the dependencies among the variables specified by the FT description do not correspond to causal relations among components of the network. Since attractor networks of the sort that realizes the neural integrator are widespread in cognitive neuroscience, the $3 \mathrm{M}$ constraint is likely to fail for a wide variety of FT cognitive models. The mechanists' requirements on genuine explanation would have the unfortunate consequence of stripping much promising research in cognitive neuroscience of its explanatory interest.

To summarize the argument in this section: many FT models fail to satisfy the mechanists' strictures on genuine explanation. They do so for one of two reasons: either (1) there is a detailed and well-confirmed account of the neural mechanism that realizes the computation, but the relation between the FT description and the realizing mechanism is not of the specific type characterized by the $3 \mathrm{M}$ constraint, viz. a mapping from arguments and values of the computed function to (states of) components of the mechanism; or (2) the neural mechanism that realizes the computation is presently unknown, though theorists may have some idea of general features of the mechanism, such as where in the brain it is located. In the second sort of case there is obviously much more theoretical work to be done. A computational model is not complete until the algorithm used to compute the function and the neural hardware that implements it have been specified. But there is no reason to think that the neural realization has to be of the specific type favored by mechanists.

In Section 5 I discuss the specific features of FT models that make them explanatory, when they are. First, though, a more general point: claims can often be explanatory in the absence of realizing details. ${ }^{14}$ That someone deliberately started a fire can be an explanation of a forest fire. Of course, it is not a complete explanation; for that we would need to know about the chemical composition of the materials involved in the incident, the condition of the prevailing winds, and so on. But, as many have noted, explanation is typically interest-relative ${ }^{15}$ sometimes the relevant interests are served without specifying the realizing details. The special sciences-including the sciences that purport to explain cognitive capacities_-are continuous with ordinary practice in this respect.

mapping between variables in the FT characterization and components of the realizing network, does not capture this sense.

${ }^{14}$ Mechanists need not deny that there can be other kinds of explanations in science. For example, Kaplan (2011, 346, fn. 14) mentions etiological causal explanations, which explain why a phenomenon occurs by citing an antecedent cause.

${ }^{15}$ See, for example, Putnam (1978), van Fraassen (1980), and Lipton (1991). 


\section{The Explanatory Credentials of Function-Theoretic Models}

Computational models are proposed to explain our manifest success at some cognitive task-seeing 3D structure in the scene, understanding speech, grasping objects in nearby space, and so on. Justifying the FT description requires explaining how computing the value of the specified function contributes to the exercise of the cognitive capacity in question. The model is answerable, primarily, to behavioral data about the performance of the system in its normal environment. Thus, the theorist's first task is to characterize, in rough and ready terms, the organism's competence in the relevant cognitive domain-in what circumstances it is successful, and in what, perhaps rare, circumstances, it fails. Often the burden will be carried not by details of the realizing neural mechanism, about which very little may be known, but by features of the environmental context in which the mechanism normally operates. As noted above, Ullman's structure-frommotion mechanism is able to recover the $3 \mathrm{D}$ structure of a moving object by computing the unique rigid interpretation consistent with three distinct views of four non-coplanar points only because in our world objects are typically rigid in translation. Appeal to a general environmental constraint (rigidity in this case) is crucial to the explanation of the organism's pattern of success and failure. Very little is known about the neural mechanism that implements the function (beyond the fact that areas V3/V3A and the dorsal parieto-occipital junction appear to be implicated).

As I have noted, an FT characterization of a cognitive mechanism resides at the topmost of Marr's explanatory levels, the so-called theory of the computation. It provides a canonical specification of the function computed by the mechanism, hence it answers a "what-question": what, precisely, does the device do? But it also takes the first step in specifying how the system computes the cognitive function that is the explanandum of the theory: it computes the cognitive function, in its normal environment, by computing the specified mathematical function. ${ }^{16}$ However, the FT characterization does not "reveal the causal structure of the mechanism," as Kaplan $(2011,352)$ requires, except at a very high level of abstraction.

By its very nature an FT characterization is multiply realizable-it subsumes both natural and artefactual computers. Moreover, much of its explanatory force depends on the fact that it is abstract. Our grasp of a mathematical characterization-say a characterization of a system as an adder or an integrator-is independent of any acquaintance we may have with particular (type or token) physical realizations of the mathematical description.

The idea that mental or other "high-level" properties are multiply realized has recently come under attack. Opponents of multiple realizability argue that only the various structure-specific realizers of putative multiply realized properties count as

\footnotetext{
16 So, schematically, a cognitive system S computes $x$ (the cognitive capacity) by computing $y$ (the mathematical function specified by FT) in context $z$ (the normal environment).
} 
genuine explanatory kinds. The dialectical context of these arguments is an attack on non-reductive materialism. Materialism (reductive or not) isn't the point here, but if these arguments succeed in undermining the integrity of multiply realized kinds then the explanatory bona fides of FT models would be threatened. Deflecting these arguments will allow me to highlight some important explanatory features of FT models.

Shapiro (2000) poses a dilemma: either the realizing kinds of a putative higher-level multiply realized property share many causally relevant properties or they do not. If the realizers share many causally relevant properties, then they are not distinct realizations. If they do not share many causally relevant properties, then any generalizations that apply to them will be "numbingly dull" $(2000,649)$. (Shapiro cites as examples of numbingly dull generalizations that all realizers of mousetraps are used to catch mice, and that camera eyes and compound eyes both have the function of facilitating sight.) So either the higher-level kind is just not multiply realized or there is no motivation for subsuming the various distinct physical kinds under a higher-level (multiply realized) kind. ${ }^{17}$

FT kinds evade both horns of the dilemma. Shapiro says "multiple realizations truly count as multiple realizations when they differ in causally relevant properties-when they make a difference to how they contribute to the capacity under investigation" (p. 644). Corkscrews that differ only in color contribute in identical ways to removing corks, but hand calculators and human brains almost certainly differ in relevant causal powers, for example, in how they contribute to the system's capacity to compute the addition function. It is very likely that they employ different algorithms that require different realizing mechanisms. But the fact that these very different physical systems both compute the addition function - the fact that we can specify their behavior over a staggeringly large range of input conditions-is hardly "numbingly dull." So Shapiro's argument fails to show that FT kinds are not genuinely multiply realized.

Klein (2008) argues that there are no cases of genuinely multiply realized kinds in science. All putative examples either only support generalizations that are projectible within the restricted-realization kind, or, if they appear to support generalizations that are projectible across other realization kinds, turn out, on closer examination, to be non-actual idealizations, and so involve no ontological commitment to the higher-level kind. Materials science provides an example of the first sort of case: it classifies as brittle various materials-brittle steel and brittle glass, for example - that otherwise have very little in common. Of all that we know about brittleness in steel-that brittleness is proportional to hardness, that steel can be made less brittle by tempering, and so onalmost nothing applies to brittle glass. Discoveries about one realization-restricted kind of brittle material are not projectible to other realization-restricted kinds. Klein goes on to say:

If there are [multiply realizable] kinds, they must be proper scientific kinds. If they are scientific kinds, then we should be able to project generalizations about them across all instances of that kind. But there aren't any such projectable discoveries; it looks like we must therefore abandon

\footnotetext{
${ }_{17}$ See Kim (1992) for a similar argument.
} 
MR kinds-and not just in metallurgy, but in all of the special sciences, and psychology in particular. (Klein 2008, 162)

Klein concludes that scientific ontologies should include only realization-restricted kinds. The upshot is that FT kinds-which appear to subsume both biological subjects and artifacts, and hence are not realization-restricted kinds-should be eliminated; at best they are idealizations that do not literally apply to anything.

I will tackle the second disjunct of the dilemma first. FT models are not idealizations, in the sense that Klein has in mind. He says:

Idealizing models do not purport to describe the world... idealizing models are mere possibilia. Talk about them is false of anything in the actual world. You can't use them to predict anything... Idealizations are typically used to explain the ceteris paribus laws that cover the (realization-restricted) kinds of particular special sciences. When scientists talk about the ideal gas, it is usually in the context of explaining the ceteris paribus laws that cover actual gasses. The ideally brittle solid is never cited on its own to explain anything. (Klein 2008, 173)

To be sure, FT characterization does involve idealization. To describe a hand calculator as computing the addition function is to attribute to it a capacity defined on an infinite domain. The calculator's actual capacity is limited by the size of its display. A similar point applies to any biological system. And artefactual and biological computers are subject to various sorts of noise. They can fail to compute the specified function when they overheat or are exposed to harmful substances. Nonetheless, the FT characterization is intended to be literally true of the calculator, as is an FT characterization of a biological system in a computational psychological model. And, as I have noted, FT characterizations allow the prediction of the device's behavior across a wide range of input conditions, viz. those corresponding to the arguments of the specified function. So they are not idealizations in Klein's sense.

The argument against the first disjunct of Klein's dilemma - that empirical discoveries about one class of realizers do not project to other classes of realizers, and so commitment to a multiply realized higher-level kind is not scientifically motivated-is somewhat less direct. It is true, of course, that what we know about the circuitry of the hand calculator is unlikely to be true of the brain (and vice versa). But the fact that lower-level physical facts about one class of realizers are not projectible to other classes is beside the point. ${ }^{18}$ The understanding we gain of the capacities of a system (whether artefactual or biological) from FT models depends on the abstract character of the capacity attributed, not on any particular physical realization of that capacity. As I noted above, this explanatory payoff of FT characterization depends on the fact that the mathematical functions deployed in computational models-addition, integration, Laplacian of Gaussians, and so on-are well understood independently of their application in such models, and independently of our familiarity with computing devices, which of course is a relatively

${ }^{18}$ Facts about the behavior of the system, under interpretation, are projectible. 
recent development. If this is right then Klein's case for the elimination of multiply realized kinds does not apply to FT kinds.

The upshot is that FT kinds are genuinely multiply realized; in fact they may be sui generis multiply realized kinds. They are not only abstract, but they are also normative. Theories of cognition are charged with explaining not just behavior, but, more importantly, cognitive capacities or competences, and FT models do so by positing further (mathematical) competences. In attributing a competence to a physical system-to add, to compute a displacement vector, and so on-FT models support attributions of correctness and mistakes. Just as the normal functioning of the system-correctly computing the specified mathematical function-explains the subject's success at a cognitive task in its normal environment, so a malfunction explains its occasional failure. Ingesting too much alcohol can cause neural systems to malfunction in any number of ways; one effect is that computational mechanisms may not compute their normal functions. One's hand overshooting the cup because the motor control system miscalculated the difference vector is a perfectly good explanation of a motor control failure. ${ }^{19}$

This gives the FT characterization a kind of autonomy - the physical description that specifies the realizing neural mechanism does not allow the reconstruction of the normative notions of correctness and mistake. ${ }^{20}$ The FT characterization explains the cognitive capacity by appeal to another competence not recoverable from the physical/ causal details alone. But though the normativity inherent in the FT description cannot be accounted for at the level of realizing mechanisms there is nothing mysterious here. Look again at the adder depicted in Figure 7.1. The bottom span of the figure specifies the physical state transitions that characterize the normal operation of the mechanism. When conditions are not normal-for example, when a human subject containing the neural adder is drunk, or a hand calculator is immersed in water-these physical state transitions may be disrupted. In other words, the system may be in the physical state(s) that (under the interpretation imposed by the mapping) realizes the arguments of the function, but fail to go into the physical state that (under interpretation) realizes the value of the function. The specification of physical state transitions (the bottom span of Figure 7.1) does not support attributions of correctness or mistake; the normative attributions are a consequence of the computational interpretation imposed by the mapping to the function.

In summary, the fact that FT characterizations are both abstract and normative (in the above sense) explains how FT models can be genuinely explanatory even absent an account of their neural realization.

\footnotetext{
${ }^{19}$ Of course, the hand may have overshot the cup for a variety of other reasons. A spasm in the arm muscles would be a different kind of malfunction.

${ }^{20}$ This is not to deny that accounts of neural mechanisms may advert to such normative notions as wellfunctioning and malfunction. But physical/causal descriptions, even when they advert to functional notions, do not support attributions of correctness and mistake. They do not allow us to say that the mechanism miscalculates (or misrepresents) the vector from hand to cup.
} 


\section{Objections and Replies}

I conclude by considering some objections.

Objection (1): FT models are not genuinely explanatory; they are what Craver (2006) and Kaplan (2011) call phenomenological models (p-models). P-models "provide descriptions (often highly abstract, mathematical descriptions) of the phenomena for which explanations are sought... [but they] merely 'save the phenomena' to be explained" (Kaplan 2011, 349).

Reply: FT characterizations are not p-models-they do not just give a mathematical description of an observed regularity; rather they claim that the device computes a particular mathematical function and in so doing produces the observed regularity. This distinction is important. The motion of the planets can be described (mathematically) by Kepler's laws, but the planets do not compute Kepler's laws, in the intended sense. The explanandum of a computational cognitive theory is a manifest cognitive capacity. An FT model is a hypothesis about how the system does it, by exercising a mathematical competence. The solar system has no manifest cognitive capacities that require appeal to mathematical competence. The objection that computational models, in the absence of realizing neural details, are just p-models, in other words that they are just descriptions of behavior, rests on a misconstrual of these models. The models give an abstract characterization of a mechanism that produces the phenomena by computing a mathematical function. ${ }^{21}$

Objection (2): FT models do not describe "the real components, activities, and organizational features of the mechanism that in fact produces the phenomena" (Craver 2006, 361). They are mere "how-possibly" models, rather than "how-actually" models. As Kaplan puts it "the cost imposed by departing from [the mechanists'] view... is the loss of a clear distinction between computational models that genuinely explain how a given phenomenon is actually produced versus those that merely describe how it might possibly be produced" $(2011,346)$.

Reply: I doubt that there is a sharp distinction between how-actually and how-possibly models. Weiskopf (2011) argues, convincingly to my mind, that the distinction is epistemological. As a model that purports to explain a given phenomenon is more fully developed and acquires additional empirical support it will typically cross the threshold from "how-possibly" to "how-actually," though the latter verdict is always defeasible. But putting aside what kind of distinction this is, FT models are hypotheses about how a system in fact exercises a particular cognitive capacity. A well-confirmed account of the algorithm used to compute the specified function and the neural structure that

${ }^{21}$ Putnam (1988) and Searle (1993) argue that every physical system computes every function. If every physical system does compute every function, in the sense at work in function-theoretic explanation, then the distinction between a system being merely describable mathematically and a system actually computing a mathematical function collapses, and computational models cannot be genuinely explanatory. The Putnam/ Searle arguments have been widely discussed. For recent responses see Chalmers (2012), Egan (2012), and the other papers in the Journal of Cognitive Science, Vol. 12. 
realizes the computation would, of course, increase our confidence that the model describes how the brain actually does it.

It should also be emphasized that FT characterizations in the first instance are specifications of the function that the device in fact computes; they are, one might say, what-explanations. Sometimes the characterization takes the form of a specification of an algorithm, in other words, an intensional specification of the function computed. In such cases, the algorithm is offered, not as an account of how possibly the device computes the function, but of what it computes and how in fact it computes it, as is evidenced by the fact that theorists would change the hypothesized algorithm were evidence to become available showing that the device is computing a function other than the one specified by the algorithm. Initial hypotheses regarding the functions computed and the algorithms for computing these functions are selected from the computational theorist's toolbox, but that fact does not undermine the claim that these are hypotheses about what the device actually does and how it actually does it. How else are theorists supposed to develop theories except by using what they know? These initial hypotheses will often be modified in light of new behavioral data, sometimes to the point that eventually the device is said to compute a function sufficiently different from the well-understood function with which the theorist began that it can only be described in task-specific intensional terms. ${ }^{22}$

Objection (3): FT characterizations are just mechanism sketches. They derive their explanatory force in the same way that other mechanistic models do, by specifying the underlying mechanism. In this case, the specification is only partial.

Reply: FT characterizations are descriptions of cognitive mechanisms. Since they do not fully specify how a cognitive mechanism works, we might call them "mechanism sketches." In any event, construing FT models as mechanism sketches is dispositive only if a mechanism sketch is explanatory just to the extent that it issues a promissory note for a decompositional mechanistic analysis of the sort specified by the $3 \mathrm{M}$ constraint. I have argued that the explanatory credentials of an FT model do not depend on the existence of a mapping that satisfies the $3 \mathrm{M}$ constraint, but rather on the FT model providing a canonical specification of the function computed by a cognitive mechanism, a crucial first step in an explanation of how the mechanism enables the cognitive capacity to be explained. Moreover, the distinctive features of FT modelstheir abstract and normative character-are not recoverable from a specification of their neural realization, even in cases where the specification does satisfy the $3 \mathrm{M}$ constraint. An account of the realizing neural architecture would, of course, increase the probability that a given FT model is true, but it is not the source of the model's explanatory force. If FT models are mechanism sketches then some mechanism sketches derive their explanatory force non-mechanistically.

22 The computational model of natural language processing developed by Marcus (1980) is a case in point: the proposed model is an augmentation of a standard $\operatorname{LR}(\mathrm{k})$ parser of the sort that one might encounter in a graduate-level course in parsing theory. The augmentations are dictated by observed features of human linguistic competence, and the resulting model can be characterized only intensionally. 
Objection (4): You say that FT characterization is autonomous. Isn't this just an expression of what Piccinini (2006) has called computational chauvinism, the idea that (as Johnson-Laird 1983, 9) put it: "[t]he mind can be studied independently from the brain... [that] psychology (the study of the programs) can be pursued independently from neurophysiology (the study of the machine and the machine code)."

Reply: No, it isn't computational chauvinism. I have explained the sense in which the FT level is autonomous-it characterizes the physical system in abstract terms, as a member of a well-understood class of mathematical devices. Moreover, the FT characterization is normative in a particular sense-it supports attributions of correctness and mistakes, notions not available at the level of realizing neural mechanisms. I am not claiming that we can fully explain cognition without understanding these neural mechanisms; in fact, I insist that we cannot. The full explanation requires both an account of the realizing mechanism (though, as I have argued, the relation between the FT characterization and the realization may not satisfy the $3 \mathrm{M}$ constraint) and, typically, an account of the environment in which the cognitive capacity is exercised. The point is rather that claims of the sort "the hand overshot the cup because the system miscalculated the difference vector" enjoy a sort of explanatory autonomy from the realization details.

\section{Postscript: Personal and Subpersonal Capacities}

My account of FT explanation refers to two kinds of capacities or competences; it is useful to clarify the relationship between the two. Cognitive capacities that are the explananda of the cognitive sciences-seeing what is where, understanding speech, reaching, and pointing-are personal-level capacities. They are achievements of the organism, things at which we are generally successful. Personal-level cognitive capacities, manifest in our behavior, are explained by positing subpersonal mechanisms that have mathematical capacities. It is something of a "category mistake" (as philosophers used to say) to say that we compute the Laplacian of a Gaussian or integration. Rather, mechanisms in our brains do this, and by doing so (in normal conditions) they enable us to see, understand speech, manipulate objects, and so on.

I have argued elsewhere (see Egan 2014) that the main job of representational content is to connect the subpersonal mechanisms characterized in abstract terms by cognitive scientific theories with the manifest personal-level capacities that it is the job of these theories to explain. Marr construes the inputs to the Laplacian/Gaussian filter as representing light intensities and outputs as representing discontinuities of light intensity. Shadmehr and Wise construe inputs and outputs of the mechanisms they posit as representing positions of objects in nearby space and changes in joint angles. In general, the inputs and outputs of FT mechanisms are characterized not only in abstract terms, as the arguments and values of the specified mathematical function; they are often characterized as representing properties or objects relevant to the cognitive capacity to be explained. Characterizing the subpersonal mechanism in terms congruent with the 
way we think about the personal-level capacity that the mechanism subserves allows us to see how the exercise of the mathematical competence contributes to our success at these tasks. Representational content is the "connective tissue" linking the subpersonal capacities posited in the theory and the manifest personal-level capacities (the "phenomena") that the theory attempts to explain. ${ }^{23}$

\section{References}

Amit, D. J. (1989), Modeling Brain Function: The World of Attractor Neural Networks. New York: Cambridge University Press.

Bechtel, W. (2008), Mental Mechanisms: Philosophical Perspectives on Cognitive Neuroscience. London: Routledge.

Bechtel, W. and Abrahamsen, A. (2005), "Explanation: A Mechanistic Alternative." Studies in History and Philosophy of the Biological and Biomedical Sciences 36: 421-41.

Bechtel, W. and Richardson, R. C. (1993), Discovering Complexity: Decomposition and Localization as Strategies in Scientific Research. Princeton, NJ: Princeton University Press.

Becker, W. and Klein, H. M. (1973), "Accuracy of Saccadic Eye Movements and Maintenance of Eccentric Eye Positions in the Dark.” Vision Research 13: 1021-34.

Chalmers, D. (2012), "A Computational Foundation for the Study of Cognition." Journal of Cognitive Science 12: 323-57.

Craver, C. (2006), "When Mechanistic Models Explain." Synthese 153: 355-76.

Craver, C. (2007), Explaining the Brain. Oxford: Oxford University Press.

Crick, F. (1989), “The Recent Excitement about Neural Networks." Nature 337: 129-32.

Cummins, R. (1975), “Functional Analysis.” Journal of Philosophy 72: 741-65.

Egan, F. (1995), “Computation and Content." Philosophical Review 104: 181-203.

Egan, F. (2012), "Metaphysics and Computational Cognitive Science: Let's Not Let the Tail Way the Dog." Journal of Cognitive Science 13: 39-49.

Egan, F. (2014), "How to Think about Mental Content." Philosophical Studies 170: 115-35.

Eliasmith, C. (2005), "A Unified Approach to Building and Controlling Spiking Attractor Networks." Neural Computation 17(6): 1276-314.

Eliasmith, C. and Anderson, C. (2003), Neural Engineering: Computation, Representation, and Dynamics in Neurobiological Systems. Cambridge, MA: MIT Press.

Gallistel, C. R. (1990), The Organization of Learning. Cambridge, MA: MIT Press.

Glimcher, P. W. (1999), "Oculomotor Control," in R. A. Wilson and F. C. Kiel (eds), MIT Encyclopedia of Cognitive Science. Cambridge, MA: MIT Press, 618-20.

Hess, R. F., Baker, C. L., Verhoeve, J. N., Keesey, U. T., and France, T. D. (1985), “The Pattern Evoked Electroretinogram: Its Variability in Normals and Its Relationship to Amblyopia." Investigative Ophthalmology and Visual Science 26: 1610-23.

${ }^{23}$ Thanks to David M. Kaplan, Sydney Keough, Robert Matthews, and Oron Shagrir for helpful comments on earlier versions of this chapter. Thanks also to participants at the Philosophy and the Brain workshop at the Institute for Advanced Studies at the Hebrew University of Jerusalem in May 2013, participants at the Graduate Student Spring Colloquium on Exploring the Subpersonal: Agency, Cognition, and Rationality at the University of Michigan, Ann Arbor, March 2014, and the students in my graduate seminar on psychological explanation at Rutgers in spring 2014. 
Johnson-Laird, P. (1983), Mental Models: Towards a Cognitive Science of Language, Inference and Consciousness. New York: Cambridge University Press.

Kaplan, D. (2011), "Explanation and Description in Computational Neuroscience." Synthese 183(3): 339-73.

Kaplan, D. and Craver, C. (2011), “The Explanatory Force of Dynamical and Mathematical Models in Neuroscience: A Mechanistic Perspective." Philosophy of Science 78(4): 601-27.

Kim, J. (1992), "Multiple Realization and the Metaphysics of Reduction." Philosophy and Phenomenological Research 52: 1-26.

Klein, C. (2008), "An Ideal Solution to Disputes about Multiply Realized Kinds." Philosophical Studies 140: 161-77.

Leigh, R. J. and Zee, D. S. (2006), The Neurology of Eye Movements (4th edition). New York: Oxford University Press.

Levy, A. (2013), “Three Kinds of New Mechanism.” Biology and Philosophy 28: 99-114.

Lipton, P. (1991), Inference to the Best Explanation. Oxford: Routledge.

Marcus, M. (1980), A Theory of Syntactic Recognition for Natural Language. Cambridge, MA: MIT Press.

Marr, D. (1982), Vision. San Francisco: W. H. Freeman.

Milkowski, M. (2013), Explaining the Computational Mind. Cambridge, MA: MIT Press.

Piccinini, G. (2006), "Computational Explanation in Neuroscience." Synthese 153: 343-53.

Piccinini, G. and Craver, C. (2011), "Integrating Psychology and Neuroscience: Functional Analyses as Mechanism Sketches." Synthese 183(3): 283-311.

Putnam, H. (1978), Meaning and the Moral Sciences. London: Routledge.

Putnam, H. (1988), Representation and Reality. Cambridge, MA: MIT Press.

Robinson, D. A. (1989), "Integrating with Neurons." Annual Review of Neuroscience 12: 33-45.

Searle, J. (1993), The Rediscovery of the Mind. Cambridge, MA: MIT Press.

Seung, S. H. (1996), "How the Brain Keeps the Eyes Still." Proceedings of the National Academy of Science USA 93: 13339-44.

Seung, S. H. (1998), "Continuous Attractors and Oculomotor Control." Neural Networks 11: 1253-8.

Seung, S. H., Lee, D. D., Reis, B. Y., and Tank, D. W. (2000), "Stability of the Memory of Eye Position in a Recurrent Network of Conductance-Based Model Neurons." Neuron 26: 259-71.

Shadmehr, R. and Wise, S. (2005), The Neurobiology of Reaching and Pointing: A Foundation for Motor Learning. Cambridge, MA: MIT Press.

Shapiro, L. (2000), "Multiple Realizations." Journal of Philosophy 97: 635-54.

Smolensky, P. (1988), "On the Proper Treatment of Connectionism." Behavioral and Brain Sciences 11: 1-23.

Ullman, S. (1979), The Interpretation of Visual Motion. Cambridge, MA: MIT Press.

van Fraassen, B. C. (1980), The Scientific Image. New York: Oxford.

Weiskopf, D. (2011), "Models and Mechanisms in Psychological Explanation." Synthese 183(3): 313-38.

Zipser, D. and Anderson, R. A. (1988), "A Back-Propagation Programmed Network that Simulates Response Properties of a Subset of Posterior Parietal Neurons," Nature 331: 679-88. 